\title{
Surface brightness fluctuations, tracers of stellar mass-loss?
}

\author{
Rosa A. González-Lópezlira ${ }^{1}$, Gustavo Bruzual-A. ${ }^{2}$, Stéphane \\ Charlot $^{3}$, Javier Ballesteros-Paredes ${ }^{1}$ \& Laurent Loinard ${ }^{1}$ \\ ${ }^{1}$ Centro de Radioastronomía y Astrofísica, Universidad Nacional Autónoma de México, \\ Apdo. Postal 72-3 (Xangari), Morelia, Michocán 58089, México \\ email: $r$.gonzalez@crya.unam.mx \\ ${ }^{2}$ Centro de Investigaciones de Astronomía, Apartado Postal 264, Mérida 5101-A, Venezuela \\ ${ }^{3}$ Institut d'Astrophysique de Paris, 98bis, bd Arago - 75014 Paris, France
}

\begin{abstract}
We present optical and IR integrated colors and SBF magnitudes, computed from stellar population synthesis models that include emission from the dusty envelopes surrounding mass-loosing TP-AGB stars. We explore the effects of varying the mass-loss rate by one order of magnitude around the fiducial value, modifying accordingly both the stellar parameters and the output spectra of the TP-AGB stars plus their dusty envelopes. We compare these models to optical and near-IR data of single AGB stars and Magellanic star clusters. Neither broad-band colors nor SBF measurements in the optical or the near-IR can discern global changes in the mass-loss rate of a stellar population. However, we predict that mid-IR SBF measurements can pick out such changes, and actually resolve whether a relation between metallicity and mass-loss exists.
\end{abstract}

Keywords. stars: AGB and post-AGB - stars: carbon — stars: mass loss — Magellanic Clouds - infrared: stars — stars: circumstellar matter — stars: evolution — galaxies: evolution — galaxies: stellar content

\section{Overview}

The purpose of this work is to produce population synthesis models that include different mass-loss rates in the TP-AGB. To this end, we combine stellar population evolutionary models with theoretical spectral energy distributions (SEDs) that take into account the radiative transfer in the dusty circumstellar envelopes. To begin with, the SEDs are calculated on the basis of the mass-loss rates included in the evolutionary tracks; we then produce SEDs for the same stellar types, but with mass-loss rates one order of magnitude above and below the rates in the tracks, in order to explore the whole range where massloss is observable in the optical. Finally, we confront the resulting theoretical broad-band colors and fluctuation magnitudes with optical and near-IR observations of AGB stars and Magellanic star clusters. We use a preliminary version of the Charlot \& Bruzual (2009; CB09 henceforth) simple stellar population (SSP) evolutionary synthesis models to compute isochrones in the age range from a few Myr to $14 \mathrm{Gyr}$, and metal (helium) content from $Z(Y)=0.0001(0.26)$ to $Z(Y)=0.07(0.34)$. The TP-AGB evolution of lowand intermediate-mass stars is followed according to the semi-empirical prescription of Marigo \& Girardi(2007). For each of the stellar metallicities considered, we have calculated CB09 models for four possible choices of the SED assigned to TP-AGB stars in the superwind phase: (1) the SEDs used in the BC03 and CB09 models (standard models hereafter), and (2) model spectra computed with the code DUSTY (Ivezić et al. 1999) for: (a) dusty envelopes that result from the fiducial $\dot{M}$ during these evolutionary phases, (b) dusty envelopes from $\dot{M}$ one order of magnitude above fiducial, and (c) dusty envelopes 

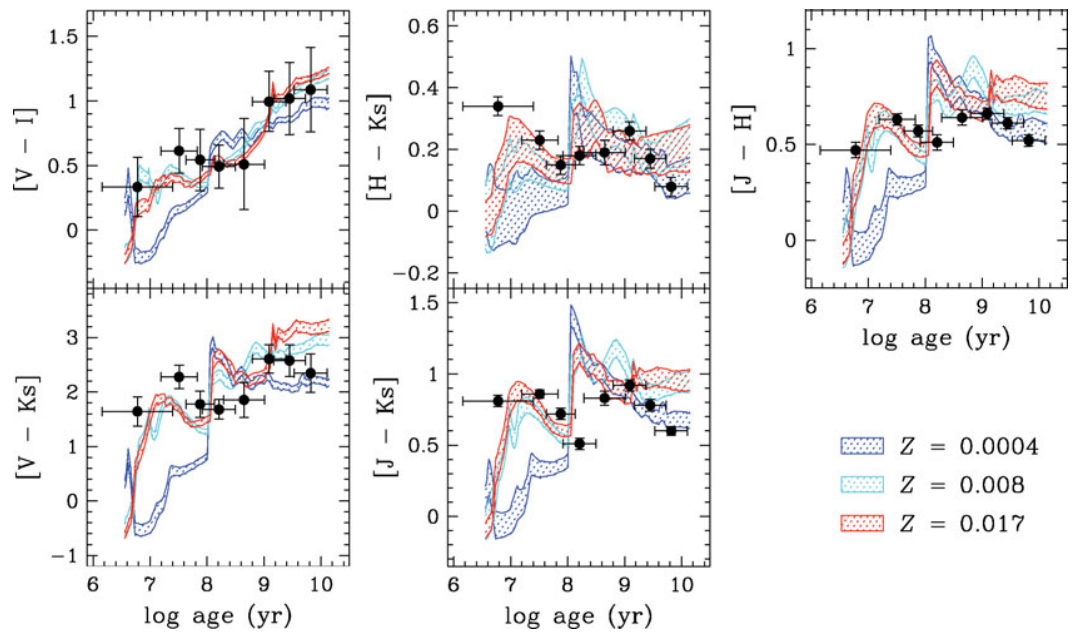

Figure 1. Colors vs. log (age); comparison between models and MC "superclusters". Colored regions represent SSPs with fiducial $\dot{M}$ and expected $1 \sigma$ error bars for $5 \times 10^{5} M_{\odot}$. Blue: $Z=$ 0.0004 ; cyan: $Z=0.008$; red: $Z=0.017$. Supercluster ages go from $6 \mathrm{Myr}$ to $10 \mathrm{Gyr}$; model ages span between $3 \mathrm{Myr}$ and $14 \mathrm{Gyr}$.

from $\dot{M}$ one order of magnitude below fiducial. The input spectra for the DUSTY code are the same spectra used at these phases by CB09. In all cases we use the Chabrier (2003) IMF. A change in $\dot{M}$, in turn, entails variations in $L$ and hence stellar lifetime, $R$, envelope and core masses, $T_{e f f}$, pulsation period, dust-to-gas ratio, dust composition, and $\mathrm{C} / \mathrm{O}$ ratio. For mass-loss rates different from the original ones in the tracks, stellar configurations and lifetimes (and hence number of stars in the phase) have been adjusted. A major challenge for this work is that we are aiming at extrapolating mass-loss rates at a large range of metallicities, but in fact a good calibration of all the parameters involved in individual TP-AGB stars exists only for Galactic and Magellanic TP-AGB stars. We venture to make predictions for lower and higher metallicities, with the clear caveat that most relations have not been tested in these conditions.

\section{Integrated colors and surface brightness fluctuations.}

We compare theoretical integrated broad-band colors to the MC clusters measured by González et al. (2004) and González-Lópezlira et al. (2005). These authors assembled 8 artificial "superclusters", by coadding data of 191 star clusters in bins with similar ages and metallicities, according to classes I - VII in the SWB categorization scheme, plus an ultra-young (pre-SWB class) supercluster. The MC "supercluster" ages, that go from $\sim 6$ Myr to $>10$ Gyr, are taken from the updated calibration by Girardi et al. (1995) of the $S$-parameter developed by Elson \& Fall $(1985 ; 1988)$; this parameter relates the ages of LMC clusters to their $U B V$ colors. Figure 1 shows the data compared to color versus age for models with a fiducial mass-loss rate. The expected $1 \sigma$ error bars for the models, shown as colored bands, have been calculated, assuming a stellar population of $5 \times 10^{5} M_{\odot} \cdot \dagger$ The trends of color with age shown by the data are very closely followed by the models.

$\dagger$ Theoretical relative errors scale as $M_{t o t}^{-1 / 2}$ (see Cerviño et al. 2002; González et al. 2004), if a Poissonian distribution is assumed for the stellar numbers in different evolutionary phases. 

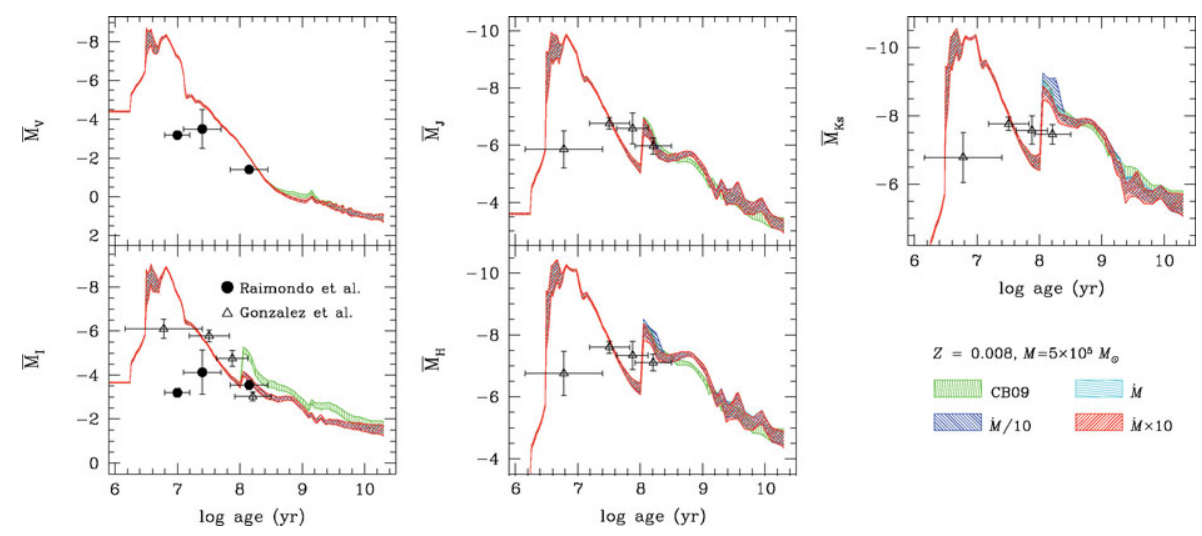

Figure 2. Model SBFs and Magellanic star cluster data. Observations of young and intermediate-age MC star clusters are compared to models with $Z=0.008$ and different mass-loss rates. Colored regions delimit expected $1 \sigma$ stochastic errors for stellar populations with $5 \times 10^{5} M_{\odot}$. Green-vertical-hatched: standard CB09; blue-left-hatched: fiducial $\dot{M} / 10 ;$ cyan-horizontal-hatched: fiducial $\dot{M}$; red-right-hatched: fiducial $\dot{M} \times 10$. Solid circles are $\bar{M}_{V}$ and $\bar{M}_{I}$ measurements for globular clusters from Raimondo et al. (2005); empty triangles are $\bar{M}_{I}$ and near-IR SBF measurements for artificial MC "superclusters" (see González et al. 2004; 2005).
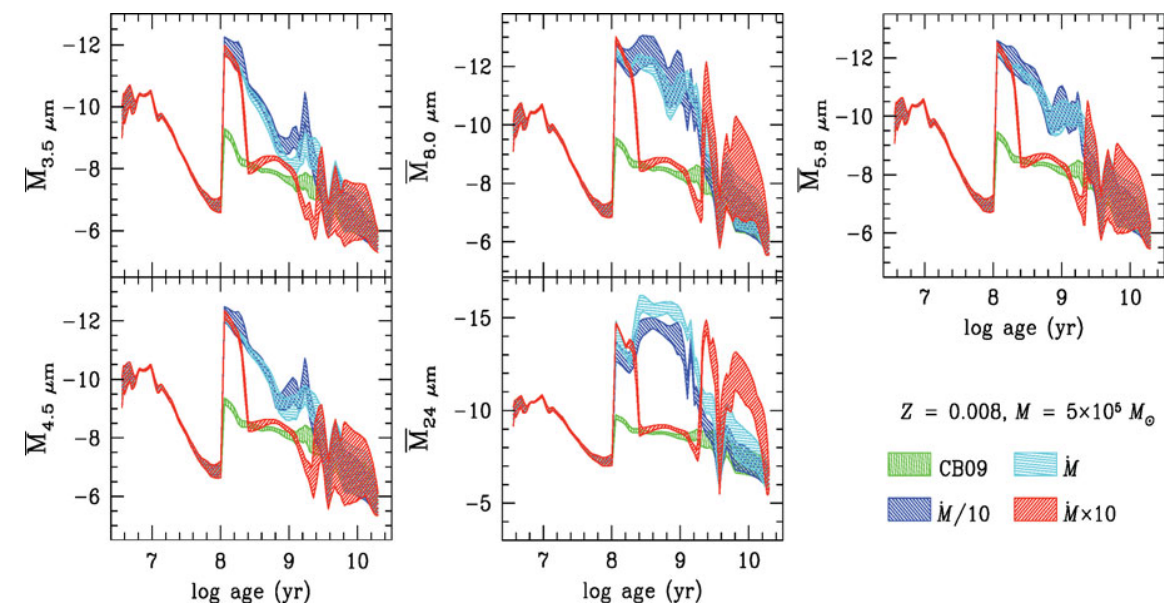

Figure 3. Mid-IR absolute fluctuation magnitudes vs. log (age) for $Z=0.008$; models with different mass-loss rates. Coloured regions, coded as in Figure 2, delimit expected $1 \sigma$ stochastic errors for stellar populations with $5 \times 10^{5} M_{\odot}$.

Figure 2 displays absolute fluctuation magnitudes vs. log (age) for models with $Z=$ 0.008 and different mass-loss rates. This metallicity $\left(Z_{\odot} / 2.1\right)$ is closest to that of the youngest half of the MC star clusters whose data are also presented. Colored regions delimit expected $1 \sigma$ stochastic errors for a stellar population with $5 \times 10^{5} M_{\odot}$. We find no strong trend of surface brightness fluctuation (SBF) brightness with mass-loss rate; the dusty models basically all fall on top of each other. Other metallicities show almost exactly the same behavior. The reason for this degeneracy is that selection effects are intensified by extinction, and further exacerbated when the mass-loss rate is changed. The probability of detecting the effects of stars shedding their envelopes in an exponential fashion, already low owing to the short duration of the phase, will decrease if the stars are heavily dust-enshrouded. If the mass-loss-rate is modified upward, the intrinsic luminosity 
of stars in the superwind stages will increase, but their lifetimes in the phase will hence be even shorter and they will be more obscured. The stars loosing the most mass sometimes will not survive past the first or second superwind stages. Contrariwise, if mass-loss rate is changed downward, stars will last longer and be less obscured, but their luminosity will go correspondingly down.

\section{Conclusions}

So, can SBF measurements at all, with their sensitivity to the brightest stars of a population, provide some insight about the mass-loss parameters of unresolved stellar populations? Figure 3 shows absolute fluctuation magnitudes vs. $\log$ (age), again for $Z=$ 0.008 , but this time in the mid-IR bands observed by the Spitzer Space Telescope. According to this figure, in the mid-IR one could begin to distinguish intermediate age stellar populations with different mass-loss rates, to the point that it might be worthwhile to start exploring the effects of other dust mixtures and even of the dust chemistry in the stellar envelopes on the integrated properties of stellar populations.

\section{References}

Cerviño, Valls-Gabaud, Luridiana, \& Mas-Hesse, J. M. 2002, A\&A, 381, 51

Chabrier, G. 2003, PASP, 115, 763

Elson, R. A. W. \& Fall, S. M. 1985, ApJ, 299, 211 $.1988, A J, 96,1383$

Girardi, L., Chiosi, C., Bertelli, G., \& Bressan, A. 1995, A\&A, 298, 87

González, R. A., Liu, M. C., \& Bruzual A., G. 2004, ApJ, 611, 270

González-Lópezlira, R. A., Albarrán, M. Y., Mouhcine, M., Liu, M. C., Bruzual-A., G., \& de Batz, B. 2005, MNRAS, 363, 1279

Ivezić, Z., Nenkova, M., \& Elitzur, M. 1999, User Manual for DUSTY, University of Kentucky Internal Report (http://www.pa.uky.edu/ moshe/dusty)

Marigo, P., \& Girardi, L. 2007, A\&A, 469, 239

Piovan, L., Tantalo, R., \& Chiosi, C. 2003, A\&A A, 408, 559

Raimondo, G., Brocato, E., Cantiello, M., \& Capaccioli, M. 2005, AJ, 130, 2625

Srinivasan, S., et al. 2009, AJ, 137, 4810 